\title{
Comprehensive Versus Standard Care in Post- Severe Acute Kidney Injury Survivors, A Randomized Controlled Trial
}

\section{Peerapat Thanapongsatorn}

Chulalongkorn University Faculty of Medicine

Kamolthip Chaikomon

Chulalongkorn University Faculty of Medicine

Nuttha Lumlertgul

Chulalongkorn University Faculty of Medicine

Khanitha Yimsangyad

King Chulalongkorn Memorial Hospital

Akarathep Leewongworasingh

King Chulalongkorn Memorial Hospital

Win Kulvichit

Chulalongkorn University Faculty of Medicine

Phatadon Sirivongrangson

King Chulalongkorn Memorial Hospital

Sadudee Peerapornratana

Chulalongkorn University Faculty of Medicine

Weerachai Chaijamorn

Siam University

Yingyos Avihingsanon

Chulalongkorn University Faculty of Medicine

Nattachai Srisawat ( $\nabla$ drnattachai@yahoo.com )

Chulalongkorn University Faculty of Medicine https://orcid.org/0000-0002-8544-8132

\section{Research}

Keywords: post-acute kidney injury, comprehensive care, severe AKI, critically ill patients, AKI survivors

Posted Date: June 11th, 2021

DOl: https://doi.org/10.21203/rs.3.rs-602432/v1 
License: (c) (i) This work is licensed under a Creative Commons Attribution 4.0 International License. Read Full License 


\section{Abstract}

Background: Currently, there is a lack of evidence to guide the optimal care for post-acute kidney injury (AKI) survivors. Therefore, post-discharge care by a multidisciplinary care team (MDCT) may improve these outcomes. This study aimed to demonstrate the outcomes of implementing comprehensive care by a MDCT in post-severe AKI survivors.

Methods: This study was a randomized controlled trial conducted between August 2018 to January 2021. Patients who survived severe AKI stage 2-3 were enrolled and randomized to be followed up with either comprehensive or standard care for 12 months. The comprehensive post-AKI care integrated a MDCT (nephrologists, nurses, nutritionists, and pharmacists). The primary outcome was the feasibility outcomes, comprised of the rates of loss to follow up, specialist consultation, 3-d dietary record, drug reconciliation, and drug alert rates at 12 months. Secondary outcomes included major adverse kidney events, estimated glomerular filtration rate (eGFR), and amount of albuminuria at 12 months.

Results: Ninety-eight AKI stage 3 survivors were enrolled, and randomized into comprehensive care and standard care groups (49 patients in each group). Compared to the standard care group, the comprehensive care group had significantly better in feasibility outcomes; higher rates of specialist consultation, 3-d dietary record, drug reconciliation, and drug alerts $(p<0.001)$. The mean eGFR at 12 months were comparable between the two groups ( 66.74 vs. $\left.61.12 \mathrm{~mL} / \mathrm{min} / 1.73 \mathrm{~m}^{2}, \mathrm{p}=0.54\right)$. The urine albumin: creatinine ratio (UACR) was significantly lower in the comprehensive care group (36.83 vs. $177.70 \mathrm{mg} / \mathrm{g}, \mathrm{p}=0.036$ ), while the blood pressure control was also better in the comprehensive care group $(87.9 \%$ vs. $57.5 \%, p=0.006)$. There was no difference in the other renal outcomes between the two groups.

Conclusions: Comprehensive care by a MDCT is feasible and could be implemented for post-severe AKI survivors. These resulted in a better reduction in the UACR and better blood pressure control.

Trial registration: clinicaltrial.gov: NCT04012008 (First registered July 9, 2019)

\section{Introduction}

Acute kidney injury (AKI) is estimated to occur in $7-18 \%$ of patients in hospital, and approximately $50 \%$ of patients admitted to the intensive care unit $(I C U)(1,2)$. Moreover, AKI survivors are at an increased risk of chronic kidney disease (CKD), end-stage renal disease, progression of albuminuria, and mortality (36). Post-severe AKI survivors also have a higher risk of heart failure, major atherosclerotic cardiovascular disease events, and all-cause death besides poor renal outcomes (7-10). These incidents are more severe in resource-limited settings. In Thailand, AKI in patients admitted to the ICU occurred in 2471 of 4688 patients (52.9\%), of which primarily stage $3 \mathrm{AKI}$ accounted for about $28.9 \%$ of them. Moreover, only $29 \%$ of AKI patients had renal recovery at the time of hospital discharge (11). 
Many AKI survivors were discharged with different degrees of renal function and renal recovery (12). Currently, a lack of evidence exists to guide the timing, frequency, and methods to evaluate kidney function and prevent poor outcomes among patients following an episode of severe AKI. In CKD patients, there have been many impressive results from the use of a multidisciplinary care team (MDCT) to improve the renal outcomes and mortality (13-17). Interestingly, AKI survivors with a nephrologists follow-up had an improved all-cause mortality (18). However, no study has so far demonstrated the benefit of implementation of a MDCT for post-severe AKI survivors. This study aimed to demonstrate the feasibility and outcomes of implementing comprehensive care in post-severe AKI survivors.

\section{Materials And Methods}

\section{Trial design and oversight}

The study was a prospective open-label randomized control trial (RCT) at King Chulalongkorn Memorial Hospital (KCMH) in Thailand from August 2018 to February 2021. The trial was registered at clinicaltrial.gov (NCT04012008) and was approved by the institutional review board at KCMH (IRB No. 005/62). The investigators informed patients or their surrogates concerning the study orally and written informed consent was given before entry into the study.

\section{Participants}

The inclusion criteria were all adult patients (age $\geq 18$ y old ) who had survived from AKI stage 2-3, defined as The Kidney Disease: Improving Global Outcomes (KDIGO) 2012 classification (19). The exclusion criteria were end-stage kidney disease patients who were receiving chronic renal replacement therapy (RRT) or having an estimated glomerular filtration rate (eGFR) of less than $15 \mathrm{~mL} / \mathrm{min} / 1.73 \mathrm{~m}^{2}$ using the CKD-EPI Creatinine Eq. (2009) prior the admission, kidney transplantation patients, moribund patients whose survival to 1 month was unlikely due to an uncontrollable comorbidity (i.e., end-stage liver or heart disease, untreatable malignancy), and patients who issued the desire not to be included in a follow-up.

\section{Randomization and intervention}

Patients were recruited and randomized at the hospital discharge in a 1:1 ratio (configured by web-based block randomization) and were stratified by their dialysis-dependent status at discharge to receive postAKI follow-up with either comprehensive or standard care for 12 months. The first visit in each group was within 4 weeks after discharge, depending on the severity of the AKI, dialysis status, and renal recovery status at discharge. Patients with dialysis-requiring AKI or non-recovery AKI were followed up within 1-2 weeks post-discharge, while patients with renal recovery were followed up at 2-4 weeks post-discharge. All of the patients were then scheduled at the post-AKI clinic every 3 months thereafter until the end of the study at 12 months (a total of five visits). To avoid contamination between the two groups, we appointed patients to attend on different days. Similarly, to minimize any ascertained bias, we used the same document format and laboratory protocol in both groups. Every patient visit in both groups had a clinical 
assessment, a routine laboratory, and intervention consisted of a renal function test by serum creatinine and urine albumin: creatinine ratio (UACR), blood chemistry, blood sugar, lipid profile, and 24-h urine output analysis to calculate the dietary protein intake and dietary salt intake, blood pressure measurement (BPM), and quality of life (QOL) measurement using the EQ-5D-5L index scores (20). However, the QOL was only measured at the first visit, and then at 6- and 12-months follow-up.

The MDCT who followed up patients in the comprehensive care groups consisted of nephrologists, renal nurses, renal pharmacists, and nutritionists. In the comprehensive care group, we focused on the process of care to improve renal and non-renal outcomes. Nephrologists were the principal physicians providing co-ordination with the other MDCT members to manage all transition care post-AKI, such as prevention of CKD progression, preparation of renal replacement therapy (RRT), or conservative therapy. Nephrologists were also responsible for dialysis related conditions, such as dialysis prescription, vascular access preparation, and dry weight adjustment; and medical related conditions, such as blood pressure control, blood sugar control, anemia management, bone and mineral disease management, volume management, cardiovascular risk management, etc.

The renal pharmacist took an essential role in documenting the drug reconciliation, which provided the details of the medication that the patients had been taking, and alert the nephrologists (drug alert) when they found a potential harmful medication or conflict or discrepancy, such as dosing error, omission, duplications, drug interaction, or nephrotoxins. This process was documented in the medication reconciliation sheet for every visit, and then scanned to the electronic medical record (EMR). Similarly, drug alerts were also recorded in the EMR. The pharmacists also helped in medication education regarding medication contribution to $\mathrm{AKI}$, such as nonsteroidal anti-inflammatory agents or antibiotics, pre-operative medication management, and adjustment of the medication dosage based on the renal function.

The renal nutritionists provided individualized dietary and nutritional counseling about the intake levels of calories, protein, sodium, potassium, phosphorus, and fluid to prevent CKD progression, dyskalemia, hyperphosphatemia, and hypervolemia based on recent nutrition guidelines (21-23). For nutritional evaluation, on every visit the nutritionist documented the nutrition assessment using the Nutrition Alert Form (NAF) and monitored dietary compliance by documenting a 3-d dietary recall, defined as the details of food consumed over the previous $3 \mathrm{~d}$.

Additionally, the nurses acted as the coordinator between the patients and the MDCT. The nurses appointed the patients to the clinic, encouraged patients to visit the clinic, and contacted patients with the team by phone or the LINE application.

Moreover, our MDCT provided knowledge of every visit to the patients about how to prevent CKD progression, dialysis education, lifestyle modification, exercise, smoking cessation, etc. We also emphasized the patients to recorded home $(\mathrm{H}) \mathrm{BPM}$, and self-monitoring blood glucose and then send the information to the LINE group (LINE application), which directly contacted with our MDCT, so the MDCT could adjust the medicine or recommendations to the patients. 
Parenthetically, only internists were in charge of following up the patients in the standard care group. The internists managed the medical condition, including the blood pressure control, blood sugar control, anemia management, bone and mineral disease management, volume management, cardiovascular risk management, as per the nephrologists. However, for patients who required dialysis or impending dialysis, the nephrologist consultation was allowed to manage the dialysis-related conditions. Likewise, the medical conditions mentioned above could be consulted by the nephrologists, based on the decision of the internists. All documents of the internist were recorded in the EMR, including information of the patient's education, medication use, drug reconciliation, drug alert, or nutrition recommendation by the internist.

\section{Study measurements}

Baseline demographic and biochemical data were collected from the EMR. Baseline creatinine was obtained from the most recent, lowest creatinine level at 7-365 days prior to admission from the EMR. However, for patients without a baseline creatinine or with missing data, we used back-calculation by reversing the MDRD equation using age, sex, and an assumed normal eGFR of $75 \mathrm{~mL} / \mathrm{min} / 1.73 \mathrm{~m}^{2}$ (24). As mentioned above, we used the same document format and laboratory protocol in both groups. All the documentation recorded by both the nephrologist and internists were obtained from the EMR, as well as the medication reconciliation and drug alert notifications from the pharmacists, and dietary records from the nutritionists. The BPM was performed using the mean of three automated BPM at an office visit, where the patient was seated and allowed 5 min of quiet rest before measurement. Anti-hypertensive medications, including the renin-angiotensin-aldosterone-system inhibitor (RAASI), were adjusted based on the decision of the physicians. The QOL was assessed with The Thai Version of the EQ-5D-5L Health Questionnaire) by an experienced nurse who was blinded to the study $(22,28)$.

\section{Outcomes}

The primary outcomes were the feasibility of the process of care by a MDCT. The outcomes were comprised of the five groups of: (i) the rate of loss to follow up; (ii) the specialist consultation rate, defined as any nephrologist consultation episode; (iii) the rate of 3-d dietary recall, defined as details of food consumed in the past $3 \mathrm{~d}$ documented by the nutritionists; (iv) the rate of drug reconciliation, defined as details of medication that the patients had been taking as documented by the pharmacists; and $(v)$ the rate of drug alerts, defined as any episode of pharmacists' notification of medication that potential harmful or conflict or discrepancy of medication. The outcomes of the 3-d dietary recall and drug reconciliation were counted only when there were the documentations of these processes for every visit. However, only one episode for nephrologist consultation and drug alert during the period of follow-up was counted for calculating their outcomes. Patients with loss to follow-up or death before the 12-months visit were not used in the calculation of the primary outcome.

The secondary outcomes were the renal outcomes and non-renal outcomes at 12 months. Five renal secondary outcomes were defined as follows. Firstly, major adverse kidney events (MAKE) in $365 d$ consisted of death, incident dialysis (a requirement for RRT), and persistent renal dysfunction (doubling 
of serum creatinine or an eGFR of more than $50 \%$ from the baseline) (25). Secondly, renal recovery, defined as a return of the serum creatinine to baseline or $<1.5$ times from baseline and no ongoing need for RRT or currently receiving RRT as acute kidney disease and renal recovery: consensus report of the Acute Disease Quality Initiative (ADQI) 16 Workgroup (12). Thirdly, incident CKD, defined as newly diagnosed CKD by eGFR criteria in patients with no previous history of CKD. Fourthly, the progression of CKD, defined as a change in the stage of CKD by eGFR criteria following the KDIGO 2012 classification (19). Lastly, the rate of recurrent AKI, defined as a new episode of AKI following the KDIGO 2012 classification (26).

The non-renal secondary outcomes included the rate of blood pressure control (defined as blood pressure $<140 / 90 \mathrm{mmHg}$ in non-hypertensive patients and $<130 / 80 \mathrm{mmHg}$ in previously hypertensive patients, as per the 2018 European Society of Cardiology (ESC) and the European Society of Hypertension (ESH) Guidelines for the management of arterial hypertension) (27), rate of RAASIs usage, rate of rehospitalization (defined as unplanned hospitalization), and the QOL after post-AKI follow up.

\section{Sample size calculation}

For the detection of a $10.0 \%$ difference in the MAKE between the comprehensive and standard care groups at a power of $80 \%$ and a $5 \%$ significance level based on a previous report, a total of 236 patients were calculated to be required (18). As a feasibility study, we aimed to use comprehensive care to stratify patients who followed-up with a MDCT. The trial was terminated on 31st January 2021 after recruiting 98 patients (49 in each of the comprehensive and standard care groups).

\section{Statistical analyzes}

Continuous variables are presented as the mean \pm one standard deviation (SD) in case of a normal distribution and as a median and interquartile range (IQR) in case of non-normally distributed variables. The student's t-test or Mann-Whitney test was used to analyze the differences between two continuous variables. Categorical variables were characterized by numbers with percentages and were compared using the Chi-square test or Fisher's exact test, as appropriate. All statistical analyses were performed with SPSS Version 22 software (SPSS, Chicago, IL), and figures were drawn using GraphPad Prism 8 (GraphPad Software Inc., California, USA).

\section{Results}

\section{Participants}

From August 2018 through January 2021, a total of 381 patients from the KCMH met the provisional eligibility requirement. Of these patients, 98 patients were randomized into the comprehensive and standard care groups (49 per group). In the comprehensive care group, nine patients (18.4\%) were lost to follow-up and seven (17.1\%) died before the end of the 12-month study period. While in the standard care group, there were eleven (22.4\%) and five (12.8\%) patients who lost to follow-up and died before the end of follow up, respectively. (Fig. 1). 
Baseline characteristics were similar between the two groups except that the comprehensive care group had a higher average age than the standard care group ( $69.7 \pm 13.8 y$ vs. $61.4 \pm 16.9 y, p=0.009)$

(Table 1). The most common AKI causes were sepsis (27.6 \%) and cardio renal syndrome $(25.2 \%)$. The RRT rate during admission, RRT dependence at discharge, and renal recovery rate were not significantly different between both groups. 
Table 1

Demographic, clinical, and biochemical data between comprehensive care and standard care

\begin{tabular}{|c|c|c|c|c|}
\hline Parameters & Total $(\mathrm{N}=98)$ & $\begin{array}{l}\text { Comprehensive } \\
\text { care }(\mathrm{N}=49)\end{array}$ & $\begin{array}{l}\text { Standard care } \\
(\mathrm{N}=49)\end{array}$ & $\begin{array}{l}\mathrm{P} \text { - } \\
\text { value }\end{array}$ \\
\hline Gender (Male), n (\%) & $53(54.1 \%)$ & $27(55.1 \%)$ & $26(53.1 \%)$ & 0.84 \\
\hline Age, mean (SD) & $65.5(15.9)$ & $69.7(13.8)$ & $61.4(16.9)$ & 0.009 \\
\hline \multicolumn{5}{|l|}{ Underlying disease: } \\
\hline Diabetes mellitus, n (\%) & $55(56.1 \%)$ & $29(59.2 \%)$ & $26(53.1 \%)$ & 0.54 \\
\hline Hypertension, n (\%) & $70(71.4 \%)$ & $35(71.4 \%)$ & $35(71.4 \%)$ & 1.00 \\
\hline CKD, n (\%) & $45(45.9 \%)$ & $22(44.9 \%)$ & $23(46.9 \%)$ & 0.84 \\
\hline Liver disease, n (\%) & $14(14.3 \%)$ & $6(12.2 \%)$ & $8(16.3 \%)$ & 0.56 \\
\hline Coronary artery disease, $\mathrm{n}(\%)$ & $26(26.5 \%)$ & $11(22.4 \%)$ & $15(30.6 \%)$ & 0.36 \\
\hline Congestive heart failure, $\mathrm{n}(\%)$ & $32(32.7 \%)$ & $13(26.5 \%)$ & $19(38.8 \%)$ & 0.20 \\
\hline Cerebrovascular disease, $\mathrm{n}(\%)$ & $14(14.3)$ & $10(20.4 \%)$ & $4(8.2 \%)$ & 0.08 \\
\hline Malignancy, n (\%) & $19(19.4 \%)$ & $10(20.4 \%)$ & $9(18.4 \%)$ & 0.80 \\
\hline AKI staging: & & & & 0.51 \\
\hline Stage $2 \mathrm{AKI}$ & $6(12.2 \%)$ & $4(8.2 \%)$ & $6(12.2 \%)$ & \\
\hline Stage $3 \mathrm{AKI}$ & $43(87.8 \%)$ & $45(91.8 \%)$ & $43(87.8 \%)$ & \\
\hline Cause of AKI: & & & & 0.57 \\
\hline Renal hypoperfusion & $18(18.4 \%)$ & $8(16.3 \%)$ & $10(20.4 \%)$ & \\
\hline Sepsis & $27(27.6 \%)$ & $15(30.6 \%)$ & $12(24.5 \%)$ & \\
\hline Nephrotoxic & $11(11.2 \%)$ & $6(12.2 \%)$ & $5(10.2 \%)$ & \\
\hline Cardio renal syndrome & $25(25.5 \%)$ & $9(18.4 \%)$ & $16(32.7 \%)$ & \\
\hline Obstructive uropathy & $4(4.1 \%)$ & $2(4.1 \%)$ & $2(4.1 \%)$ & \\
\hline
\end{tabular}

Significant values are shown in bold type, Data are shown as median (IQR) or mean (SD)

Abbreviations: AKI, acute kidney injury; GFR, glomerular filtration rate; ICU, intensive care unit; IQR, interquartile range; NGAL, neutrophil gelatinase-associated lipocalin; RRT, renal replacement therapy; SD, standard deviation

${ }^{\text {a}}$ Estimated GFR was calculated by the CKD-EPI creatinine Eq. (2009)

${ }^{b}$ Renal recovery was defined as the serum creatinine level had returned to baseline or $<1.5$ times from baseline and not ongoing need for RRT or currently receiving RRT. 


\begin{tabular}{|c|c|c|c|c|}
\hline Parameters & Total $(\mathbf{N}=98)$ & $\begin{array}{l}\text { Comprehensive } \\
\text { care }(\mathrm{N}=49)\end{array}$ & $\begin{array}{l}\text { Standard care } \\
(\mathrm{N}=49)\end{array}$ & $\begin{array}{l}\mathrm{P}- \\
\text { value }\end{array}$ \\
\hline Systemic disease & $8(8.2 \%)$ & $6(12.2 \%)$ & $2(4.1 \%)$ & \\
\hline Other & $5(5.1 \%)$ & $3(6.1 \%)$ & $2(4.1 \%)$ & \\
\hline RRT during admission, $\mathrm{n}(\%)$ & $70(71.4 \%)$ & $33(67.3 \%)$ & $37(75.5 \%)$ & 0.37 \\
\hline $\begin{array}{l}\text { Baseline serum creatinine } \\
(\mathrm{mg} / \mathrm{dL}) \text {, median (IQR) }\end{array}$ & $\begin{array}{l}1.25(0.88 \\
2.31)\end{array}$ & $1.31(0.89,2.42)$ & $1.25(0.80,2.18)$ & 0.62 \\
\hline $\begin{array}{l}\text { Baseline GFR }\left(\mathrm{mL} / \mathrm{min} / 1.73 \mathrm{~m}^{2}\right) \text {, } \\
\text { median (IQR) }\end{array}$ & $\begin{array}{l}51.50(22.39 \\
87.32)\end{array}$ & $\begin{array}{l}48.61(19.86 \\
82.11)\end{array}$ & $\begin{array}{l}52.90(29.18 \\
87.97)\end{array}$ & 0.46 \\
\hline $\begin{array}{l}\text { Discharge creatinine }(\mathrm{mg} / \mathrm{dL}) \text {, } \\
\text { median (IQR) }\end{array}$ & $\begin{array}{l}1.87(1.26 \\
3.31)\end{array}$ & $1.53(1.04,2.93)$ & $1.71(1.29,2.49)$ & 0.66 \\
\hline $\begin{array}{l}\text { Discharge GFR }(\mathrm{mL} / \mathrm{min} / 1.73 \\
\left.\mathrm{m}^{2}\right) \text {, median }(\mathrm{IQR})^{\mathrm{a}}\end{array}$ & $\begin{array}{l}30.80(17.45 \\
52.83)\end{array}$ & $\begin{array}{l}42.14(18.73 \\
71.90)\end{array}$ & $\begin{array}{l}32.06(21.57 \\
56.70)\end{array}$ & 0.84 \\
\hline $\begin{array}{l}\text { Baseline urine NGAL at } \\
\text { enrollment (ng/mL) median } \\
\text { (IQR) }\end{array}$ & $\begin{array}{l}472.85 \\
(193.50 \\
1686.75)\end{array}$ & $\begin{array}{l}472.85 \\
(175.68,1481.25)\end{array}$ & $\begin{array}{l}631.50 \\
(188.50,1874.25)\end{array}$ & 0.92 \\
\hline $\begin{array}{l}\text { Hospital length of stay (d), } \\
\text { median (IQR) }\end{array}$ & $16(11,31)$ & $15(11.25,30)$ & $16(11,34)$ & 0.84 \\
\hline ICU admission, n (\%) & 75 (76.5\%) & $36(73.5 \%)$ & $39(79.6 \%)$ & 0.48 \\
\hline $\begin{array}{l}\text { ICU length of stay (d), median } \\
\text { (IQR) }\end{array}$ & $8(5,13)$ & $9.5(5.75,13.5)$ & $8(4.75,13.25)$ & 0.28 \\
\hline $\begin{array}{l}\text { RRT dependence at discharge } \\
\text { date, } n(\%)\end{array}$ & $16(16.33 \%)$ & $8(16.3 \%)$ & $8(16.3 \%)$ & 1.00 \\
\hline $\begin{array}{l}\text { Renal recovery at discharge date, } \\
\mathrm{n}(\%)^{\mathrm{b}}\end{array}$ & $71(72.45 \%)$ & $33(67.3 \%)$ & $38(77.6 \%)$ & 0.37 \\
\hline \multicolumn{5}{|c|}{ Significant values are shown in bold type, Data are shown as median (IQR) or mean (SD) } \\
\hline \multicolumn{5}{|c|}{$\begin{array}{l}\text { Abbreviations: AKI, acute kidney injury; GFR, glomerular filtration rate; ICU, intensive care unit; IQR, } \\
\text { interquartile range; NGAL, neutrophil gelatinase-associated lipocalin; RRT, renal replacement therapy; } \\
\text { SD, standard deviation }\end{array}$} \\
\hline \multicolumn{5}{|c|}{ aEstimated GFR was calculated by the CKD-EPI creatinine Eq. (2009) } \\
\hline
\end{tabular}

\section{Feasibility of comprehensive care}


Patients in the comprehensive care group had a significantly higher adherence to the process of post-AKI care (Table 2), where 100\% (33 patients) of patients in the comprehensive care group received a specialist consultation by nephrologists versus $27.3 \%$ (nine patients) in the standard care group $(p<0.001)$. However, four patients (two lost to follow-up and two died) in the comprehensive care group and six patients (two lost to follow-up and four died) in the standard care group who were previously RRTdependent at discharge were excluded. The result of nutrition care by a nutritionists using a 3-d dietary recall was significantly different between patients in the comprehensive and standard care groups (100\% vs. $0 \%$, respectively, $\mathrm{p}<0.001)$, as were the pharmacists' rate of drug reconciliation and drug alert care ( $100 \%$ vs. $0 \%$ and $33.3 \%$ vs. $0 \%$, respectively, both $p<0.001)$. However, the follow-up loss rate was not significantly different between the two groups (18.4\% and $22.4 \%$ in the comprehensive and standard care groups, respectively, $\mathrm{p}=0.62$ ).

Table 2

Feasibility outcomes of the patient

\begin{tabular}{|c|c|c|c|}
\hline & Comprehensive care & Standard care & P-value \\
\hline Loss to follow up rate, $\mathrm{n}(\%)$ & $9 / 40(18.4 \%)$ & $11 / 38(22.4 \%)$ & 0.62 \\
\hline Rate of specialist consultation, $\mathrm{n}(\%)^{\mathrm{a}}$ & $33 / 33(100.0 \%)$ & 9/33 (27.3\%) & $<0.001$ \\
\hline Rate of 3-day dietary recall using, $n(\%)^{b}$ & $33 / 33(100.0 \%)$ & $0 / 33(0 \%)$ & $<0.001$ \\
\hline Rate of drug reconciliation, $\mathrm{n}(\%)^{\mathrm{c}}$ & $33 / 33(100.0 \%)$ & $0 / 33(0 \%)$ & $<0.001$ \\
\hline Rate of drug alert, $n(\%)^{d}$ & 11/33 (33.3\%) & $0 / 33(0 \%)$ & $<0.001$ \\
\hline \multicolumn{4}{|l|}{ Significant values are shown in bold type } \\
\hline \multicolumn{4}{|c|}{ aSpecialist consultation; any episode of nephrologist consultation } \\
\hline \multicolumn{4}{|c|}{${ }^{\mathrm{b}}$ Dietary recall; details of food consumed in the past three days documented by the nutritionist } \\
\hline \multicolumn{4}{|c|}{$\begin{array}{l}\text { 'Drug reconciliation; details of medication that the patients had been taking as documented by the } \\
\text { pharmacist }\end{array}$} \\
\hline
\end{tabular}

\section{Renal outcomes}

During follow-up, the comprehensive care group had a significantly reduced UACR compared to the standard group from 3 months onwards, and was lowest at 12 months ( 36.83 vs. $177.70 \mathrm{mg} / \mathrm{g}$, respectively, $p=0.036$ ) (Table 3, Fig. 2, Table S2). 
Table 3

Outcome at 12 months follow up

\begin{tabular}{|c|c|c|c|}
\hline Parameters & Comprehensive care & Standard care & P-value \\
\hline Serum creatinine $(\mathrm{mg} / \mathrm{dL}) *$ & $1.14(0.80,1.46)$ & $1.05(0.84,2.08)$ & 0.49 \\
\hline $\operatorname{eGFR}\left(\mathrm{mL} / \mathrm{min} / 1.73 \mathrm{~m}^{2}\right)^{*}$ & $66.74(30.77)$ & $61.23(35.16)$ & 0.54 \\
\hline $\operatorname{UACR}(\mathrm{mg} / \mathrm{g}) \star$ & $36.83(13.39,131.90)$ & $177.70(47.12,745.71)$ & 0.036 \\
\hline MAKE 365, n (\%) & $13 / 40(32.5 \%)$ & $11 / 38(28.9 \%)$ & 0.73 \\
\hline Death, n(\%) & $7 / 40(17.1 \%)$ & $5 / 38(12.8 \%)$ & 0.59 \\
\hline RRT, n(\%) & 6/33 (18.2\%) & $3 / 33(9.1 \%)$ & 0.28 \\
\hline Doubling serum creatinine, $\mathrm{n}(\%)$ & $1 / 33(3.0 \%)$ & 4/33 (12.1\%) & 0.36 \\
\hline Renal recovery, $\mathrm{n}(\%)^{\mathrm{b}}$ & $8 / 16(50 \%)$ & $4 / 11(36.4 \%)$ & 0.70 \\
\hline New RRT, $n(\%)^{c}$ & $1 / 28(3.6 \%)$ & $2 / 31(6.5 \%)$ & 0.62 \\
\hline New CKD, n (\%) ${ }^{d}$ & $3 / 22(13.6 \%)$ & $4 / 19(21.1 \%)$ & 0.69 \\
\hline CKD progression, $\mathrm{n}(\%)^{\mathrm{e}}$ & $7 / 11(63.6 \%)$ & $11 / 14(78.6 \%)$ & 0.41 \\
\hline Recurrent AKI, n (\%) ${ }^{f}$ & 6/33 (18.2\%) & 6/33 (18.2\%) & 1.00 \\
\hline Readmission, $\mathrm{n}(\%)^{\mathrm{g}}$ & $27 / 49(55.1 \%)$ & $24 / 49(49 \%)$ & 0.54 \\
\hline Blood pressure control, $\mathrm{n}(\%)^{\mathrm{h}}$ & 29/33 (87.9\%) & 19/33 (57.6\%) & 0.006 \\
\hline RAAS inhibitor use, n (\%) & 18/33 (54.5\%) & 14/33 (42.4\%) & 0.33 \\
\hline 24-h urine sodium (mmol) & $112.82(63.17)$ & $127.69(54.23)$ & 0.35 \\
\hline 24-h dietary protein intake $(\mathrm{g} / \mathrm{kg} / \mathrm{d})$ & $1.01(0.32)$ & $0.85(0.28)$ & 0.07 \\
\hline Albumin $(\mathrm{g} / \mathrm{dL})$ & $4.1(3.8,4.4)$ & $4.0(3.8,4.4)$ & 0.59 \\
\hline EQ-5D-5L index scores ${ }^{i}$ & $0.96(0.90,1.00)$ & $0.99(0.80,1.00)$ & 0.80 \\
\hline \multicolumn{4}{|c|}{ Significant values are shown in bold type, *Data excluded patients with RRT. } \\
\hline \multicolumn{4}{|c|}{$\begin{array}{l}\text { Abbreviations: AKI, acute kidney injury; CKD, chronic kidney disease; GFR, glomerular filtration rate; } \\
\text { RAAS, renin-angiotensin-aldosterone-system; RRT, Renal replacement therapy; SD, standard deviation; } \\
\text { UACR, urine albumin creatinine ratio }\end{array}$} \\
\hline \multicolumn{4}{|c|}{$\begin{array}{l}\text { a MAKE } 365 \text {; major adverse kidney events at } 365 \mathrm{~d} \text { were comprised of death, incident dialysis } \\
\text { (requirement for CRRT), and persistent renal dysfunction death (doubling of serum creatinine or eGFR } \\
<50 \% \text { from baseline) }\end{array}$} \\
\hline
\end{tabular}


${ }^{b}$ Renal recovery; serum creatinine level had returned to baseline or $<1.5$ times from baseline and not ongoing need for RRT or currently receiving RRT (only patients who were non-recovery at discharge)

${ }^{c}$ New RRT; new case of RRT

${ }^{d}$ New CKD; newly diagnosis of chronic kidney disease by eGFR criteria in no previous history of CKD

e CKD progression; change of staging of CKD by eGFR criteria in previous history of chronic kidney disease

${ }^{\mathrm{f}}$ Recurrent AKI; document of AKI definition by KDIGO 2012 criteria

g Readmission; episode of unplanned readmission

${ }^{h}$ Blood pressure control; blood pressure $<140 / 90 \mathrm{mmHg}$ in non-hypertensive patients or $<130 / 80$ $\mathrm{mmHg}$ in hypertensive patients

' EQ-5D-5L index scores; descriptive system for health-related quality of life states in adults, consisting of five dimensions (Mobility, self-care, usual activities, pain \& discomfort and anxiety \& depression)

There was no meaningful difference in the reduction of serum creatinine (Table S1, Figure S1), the incidence of MAKE at 365 days, and the incidence of recurrent AKI between both groups. The comprehensive care group showed a significantly higher rate of renal recovery ( $50 \%$ vs. $36.4 \%, p=0.70)$, but the numerically lower incidence of new RRT, new CKD, and CKD progression in the comprehensive care group were not statistically significant $(p=0.62,0.69$, and 0.41 respectively).

\section{Non-renal outcomes}

There was a significantly better blood pressure control in the comprehensive care group ( $87.9 \%$ vs. $57.6 \%$; $p=0.006$ ) (Table 3 ). However, the rate of RAASI use was not significantly different between the two groups $(54.5 \%$ vs. $42.4 \%$; $=0.33)$, nor was the readmission rate $(55.1 \%$ vs. $49 \%$; $p=0.54)$. The QOL (EQ$5 \mathrm{D}-5 \mathrm{~L}$ index score) tended to be better in the comprehensive care group, but this was not significant (0.99 [0.8-1.0] vs. 0.96 [0.8-1.0], $p=0.80$ ). The nutrition, as shown by the 24 -h urine sodium and protein intakes, was not significantly different between the two groups (112.82 \pm 63.17 vs. $127.69 \pm 54.23 \mathrm{mmol}$, $p=0.35$; and $1.01 \pm 0.32$ vs. $0.85 \pm 0.28 \mathrm{~g} / \mathrm{kg} / \mathrm{d}$, respectively). Other results between the two groups were not significantly different (Table S3).

\section{Discussion}

Our study was the first RCT to explore the outcomes of comprehensive care by a MDCT in patients with post-severe AKI. We demonstrated the optimal process of care for post-severe AKI survivors according to the ADQI 16 Workgroup (12) and Quality Improvement Goals for AKI (28) guidelines. Our study showed 
the successful feasibility of a MDCT in post-AKI care, with significantly better rates of specialist consultation, drug reconciliation, drug alert, and dietary recall.

Previously, post-AKI survivors were rarely followed up after discharge even though there have been many studies demonstrating impressive outcomes in patients receiving post-discharge care in other diseases, such as heart failure and myocardial infarction $(29,30)$. A previous study showed that only $8.5 \%$ of AKI survivors had nephrology referrals after discharge (31), which is consistent with another retrospective study that showed that only $12 \%$ of post-AKI patients received a specialist nephrology follow-up, while only $57 \%$ had their serum creatinine level measured within 3-6 months following discharge (32). Our study showed completely serum creatinine measurement in every visit in both comprehensive care group and standard care group, except those with loss to follow-up and dead. We also showed successful postAKI survivors follow-up by a nephrologist (100\% in a comprehensive care group and $27.3 \%$ in standard care group, $p<0.001$ ). (Table 2 ).

Moreover, post-AKI survivors had several co-morbid sequelae and may have many medication uses. These increase the risk of drug duplications, dosing error, drug interactions, or nephrotoxic drug prescription. In the comprehensive care group, we demonstrated the pharmacists' role to take responsibility for drug records, drug dosing adjustment, and drug alerts to prevent such problems that may otherwise have harmed the patients. Our study showed a significant improvement in drug reconciliation and drug alert ( $100 \%$ vs. $0 \%$ and $33.3 \%$ vs. $0 \%$, respectively, $\mathrm{p}<0.001)$. As well as CKD patients, post-AKI survivors tend to have malnutrition that is related to their QOL and renal outcomes (3335). We showed an important role of the nutritionist was to provide food information, a guideline on the number of calories, minerals, and other nutrients by recording a 3-d dietary recall, which is one of the most widely used tools in nutrition epidemiology to identify food, energy, and nutrient intake to evaluate diet assessment (36). The 3-d dietary recall rate was significantly better in the comprehensive than in the standard care group $(100 \%$ vs. $0 \%$, respectively, $p<0.001)$ (Table 2$)$.

The MDCT's effect on the comprehensive care group resulted in the numerical reduction of the UACR ( $36.83 \mathrm{mg} / \mathrm{g}$ ) compared to the standard care group (vs. $177.70 \mathrm{mg} / \mathrm{g}$ ), and this was significantly lower at 6 - and 12-months follow up after discharge ( $p=0.044$ and 0.036 , respectively), as seen in Table $S 2$ and Fig. 2. The result may be from the optimized blood pressure control in the comprehensive care groups more than the reduced rate of RAASI usage. A recent study showed that a higher UACR was associated with a higher risk of CKD progression (37). However, our study demonstrated a lower rate of CKD progression in the comprehensive care group, which could explain these hypotheses (Table 3 ).

Despite a reduced UACR, the comprehensive care did not show any significant reduction in the MAKE at $365 \mathrm{~d}$, CKD progression, new diagnosis of CKD, and renal recovery at 12 months, compared to the standard care (Table 3). However, our finding corresponded to recent trial (FUSION trial) (38), which showed comparable MAKE365 outcome between post-AKI survivors follow-up by nephrologist versus usual care, $(44 \%$ vs $43 \%, \mathrm{RR}=1.02,95 \% \mathrm{Cl} 0.6-1.73)$. 
We also reported the QOL in post-AKI survivors. The comprehensive care group tendecd to have a better QOL than the standard care group. This is an essential issue since severe AKI survivors had a lower physical and mental status than the normal population (39), so the improved QOL in these subgroups of patients could be a better outcome.

Our study has several strengths. Firstly, we demonstrated the first RCT of implementing a MDCT in postsevere AKI survivors, especially in the high risk group. Overall, $87.8 \%$ of patients had stage $3 \mathrm{AKI}$, of which $76.5 \%$ were admitted to the ICU and $71.4 \%$ received RRT. Secondly, our MDCT completed all aspected of post-AKI health care bundles, being comprised of KAMPS (Kidney function, Advocacy, Medications, Pressure, and Sick day protocol) for all patients with AKI and WATCH-ME (Weight assessment, Access, Teaching, Clearance, Hypotension, and Medications) for AKI-D patients as recently proposed in the Quality Improvement Goal for AKI (28). Thirdly, our study had the same format document and laboratory protocol in both comprehensive care group and standard care group, therefore this minimized the ascertain bias and missing data problem. Finally, our study showed impressive outcomes of comprehensive care to reduce albuminuria, which is the hallmark of CKD progression.

Nevertheless, our study had several limitations. Firstly, the comprehensive care by the MDCT may not be available for centers in a resource-limited settings. However, our study demonstated the process of care for AKI survivors which may be apllied to other available clinics such as chronic kidney disease clinics (CKD clinics) or other metaboic clinics. Those clinics usually are available in resource-limited countries. Secondly, our study was only a pilot and feasibility study, and so with the small sample sizes the renal outcomes for implementation of comprehensive care may not be seen. We have calculated the minimum sample size to show the benefit of the MDCT (reduction of MAKE) approach over the standard should be at least 312 patients for each group. Therefore, a larger population and extension of the follow-up time periods may show these benetfits. Moreover, our study showed the over-treatment in the standard care group by the internist when the nephrologist could be consulted. This may be the explanation for the nonsignificant outcomes between both groups, except for the reduced UACR and improved rate of blood pressure control.

\section{Conclusions}

Our study explored the role of comprehensive care to improve the outcomes in post-severe AKI survivors. The study showed that comprehensive care by a MDCT is feasible and can be implemented for postsevere AKI survivors. Moreover, the comprehensive care groups had better results in the reduction of UACR and blood pressure control. Further study with larger number of patients should be conducted to establish the benefit of a MDCT approach in this high risk group.

\section{Abbreviations}

ADQI: Acute Disease Quality Initiative; AKI: Acute kidney injury; BPM: Blood pressure measurement; CKD: Chronic kidney disease; eGFR: Estimated glomerular filtration rate; EMR: Electronic medical record; KCMH: 
King Chulalongkorn Memorial Hospital; KDIGO: Kidney Disease: Improving Global Outcomes; ICU: Intensive care unit; IQR: Interquartile range; MAKE: Major adverse kidney events; MDCT: Multidisciplinary care team; NGAL: Neutrophil gelatinase-associated lipocalin; QOL: Quality of life; RAASI: Reninangiotensin-aldosterone-system inhibitor; RRT: Renal replacement therapy; SD: Standard deviation; UACR: Urine albumin creatinine ratio

\section{Declarations}

Availability of data and materials

On reasonable request, data from this study are available from the corresponding author.

\section{Acknowledgements}

We thank all the investigators, The Excellent Center for Critical Care Nephrology team, and patients for participating in this study

\section{Funding}

This study is a part of a project supported by the Excellence Center for Critical Care Nephrology, KCMH, and is funded by the National Kidney Foundation of Thailand.

\section{Author Information}

\section{Affiliations}

1. Division of Nephrology, Department of Medicine, Faculty of Medicine, Chulalongkorn University, Bangkok, Thailand

Peerapat Thanapongsatorn, Nuttha Lumlertgul, Win Kulvichit, Sadudee Peerapornratana, Yingyos Avihingsanon \& Nattachai Srisawat

\section{Excellence Center for Critical Care Nephrology, King Chulalongkorn Memorial Hospital, Bangkok, Thailand}

Peerapat Thanapongsatorn, Nuttha Lumlertgul, Khanitha Yimsangyad, Akarathep Leewongworasingh, Sadudee Peerapornratana \& Nattachai Srisawat

\section{Lerdsin Hospital, Bangkok, Thailand}

Kamolthip Chaikomon 
4. Critical Care Nephrology Research Unit, Faculty of Medicine, Chulalongkorn University, Bangkok, Thailand

Nuttha Lumlertgul, Sadudee Peerapornratana \& Nattachai Srisawat

5. Department of Medicine, Somdech Phra Pinklao Hospital, Bangkok, Thailand

Phatadon Sirivongrangson

6. Department of Laboratory Medicine, Faculty of Medicine, Chulalongkorn University, Bangkok, Thailand

Sadudee Peerapornratana

7. Faculty of Pharmacy, Siam University, Bangkok, Thailand

Weerachai Chaijamorn

\section{Academy of Science, Royal Society of Thailand, Bangkok, Thailand}

Nattachai Srisawat

\section{Authors' contributions}

$P T, K C, N L$, and NS were responsible for study concept and design. PT, KC, NL, KY, AL, WK, PS, WC, YA, and NS were responsible for the acquisition, analysis, or interpretation of data. PT and NS were responsible for drafting the manuscript. PT and NS were responsible for statistical analysis. All authors had full access to all the data in the study and take responsibility for the integrity of the data and the accuracy of the data analysis. All authors interpreted the findings, contributed to writing the manuscript, and approved the final version for publication.

\section{Corresponding author}

Correspondence to Nattachai Srisawat, MD, PhD.

\section{Ethics declarations}

\section{Ethics approval and consent to participate:}

The trial was approved by the institutional review board at KCMH (IRB No. 005/62). The investigators informed patients or their surrogates concerning the study orally and written informed consent was given before entry into the study.

\section{Consent for publication}

Not applicable. 


\section{Competing interests}

The authors declare that they have no competing interests.

\section{References}

1. Susantitaphong P, Cruz DN, Cerda J, et al. World incidence of AKl: a meta-analysis [published correction appears in Clin J Am Soc Nephrol. 2014 Jun 6;9(6):1148]. Clin J Am Soc Nephrol. 2013;8(9):1482-1493.

2. Singbartl K, Kellum JA. AKI in the ICU: definition, epidemiology, risk stratification, and outcomes. Kidney Int. 2012;81(9):819-25.

3. Lafrance JP, Miller DR. Acute kidney injury associates with increased long-term mortality. J Am Soc Nephrol. 2010;21(2):345-52.

4. Chawla LS, Amdur RL, Amodeo S, Kimmel PL, Palant CE. The severity of acute kidney injury predicts progression to chronic kidney disease. Kidney Int. 2011;79(12):1361-9.

5. Chawla LS, Eggers PW, Star RA, Kimmel PL. Acute kidney injury and chronic kidney disease as interconnected syndromes. N Engl J Med. 2014 Jul;3(1):58-66. 371(.

6. Coca SG, Singanamala S, Parikh CR. Chronic kidney disease after acute kidney injury: a systematic review and meta-analysis. Kidney Int. 2012;81(5):442-8.

7. Linder A, Fjell C, Levin A, Walley KR, Russell JA, Boyd JH. Small acute increases in serum creatinine are associated with decreased long-term survival in the critically ill. Am J Respir Crit Care Med. 2014;189(9):1075-81.

8. Bansal N, Matheny ME, Greevy RA Jr, et al. Acute Kidney Injury and Risk of Incident Heart Failure Among US Veterans. Am J Kidney Dis. 2018;71(2):236-45.

9. Go AS, Hsu CY, Yang J, et al. Acute Kidney Injury and Risk of Heart Failure and Atherosclerotic Events. Clin J Am Soc Nephrol. 2018;13(6):833-41.

10. Odutayo A, Wong CX, Farkouh M, et al. AKI and Long-Term Risk for Cardiovascular Events and Mortality. J Am Soc Nephrol. 2017;28(1):377-87.

11. Srisawat N, Kulvichit W, Mahamitra N, et al. The epidemiology and characteristics of acute kidney injury in the Southeast Asia intensive care unit: a prospective multicentre study. Nephrol Dial Transplant. 2020;35(10):1729-38.

12. Chawla LS, Bellomo R, Bihorac A, et al. Acute kidney disease and renal recovery: consensus report of the Acute Disease Quality Initiative (ADQI) 16 Workgroup. Nat Rev Nephrol. 2017;13(4):241-57.

13. Bayliss EA, Bhardwaja B, Ross C, Beck A, Lanese DM. Multidisciplinary team care may slow the rate of decline in renal function. Clin J Am Soc Nephrol. 2011;6(4):704-10.

14. Wang SM, Hsiao LC, Ting IW, et al. Multidisciplinary care in patients with chronic kidney disease: A systematic review and meta-analysis. Eur J Intern Med. 2015;26(8):640-5. 
15. Chen PM, Lai TS, Chen PY, et al. Multidisciplinary care program for advanced chronic kidney disease: reduces renal replacement and medical costs. Am J Med. 2015;128(1):68-76.

16. Hsu HT, Chiang YC, Lai YH, Lin LY, Hsieh HF, Chen JL. Effectiveness of Multidisciplinary Care for Chronic Kidney Disease: A Systematic Review. Worldviews Evid Based Nurs. 2021;18(1):33-41.

17. Nicoll R, Robertson L, Gemmell E, Sharma P, Black C, Marks A. Models of care for chronic kidney disease: A systematic review. Nephrology (Carlton). 2018;23(5):389-96.

18. Harel Z, Wald R, Bargman JM, et al. Nephrologist follow-up improves all-cause mortality of severe acute kidney injury survivors. Kidney Int. 2013;83(5):901-8.

19. KDIGO CKD Work Group. KDIGO 2012 clinical practice guideline for the evaluation and management of chronic kidney disease. Kidney Int Suppl. 2013;3:1-150.

20. Pattanaphesaj J. Health-related quality of life measure (EQ-5D-5L): measurement property testing and its preference-based score in Thai population [Doctoral dissertation]: Mahidol University; 2014.

21. Ikizler TA, Burrowes JD, Byham-Gray LD, et al. KDOQI Clinical Practice Guideline for Nutrition in CKD: 2020 Update [published correction appears in Am J Kidney Dis. 2021 Feb;77(2):308]. Am J Kidney Dis. 2020;76(3 Suppl 1):S1-S107.

22. Fiaccadori $E$, Sabatino A, Barazzoni R, et al. ESPEN guideline on clinical nutrition in hospitalized patients with acute or chronic kidney disease. Clin Nutr. 2021;40(4):1644-68.

23. Kalantar-Zadeh K, Fouque D. Nutritional Management of Chronic Kidney Disease. N Engl J Med. 2017;377(18):1765-76.

24. Thomas ME, Blaine $C$, Dawnay $A$, et al. The definition of acute kidney injury and its use in practice. Kidney Int. 2015;87(1):62-73.

25. Billings FT 4th, Shaw AD. Clinical trial endpoints in acute kidney injury. Nephron Clin Pract. 2014;127(1-4):89-93.

26. Khwaja A. KDIGO Clinical Practice Guidelines for Acute Kidney Injury. Nephron Clin Pract. 2012;120:c179-84.

27. Williams B, Mancia G, Spiering W, et al. 2018 ESC/ESH Guidelines for the management of arterial hypertension [published correction appears in Eur Heart J. 2019 Feb 1;40(5):475]. Eur Heart J. 2018;39(33):3021-3104.

28. Kashani K, Rosner MH, Haase M, et al. Quality Improvement Goals for Acute Kidney Injury. Clin J Am Soc Nephrol. 2019;14(6):941-53.

29. Ezekowitz JA, van Walraven C, McAlister FA, Armstrong PW, Kaul P. Impact of specialist follow-up in outpatients with congestive heart failure. CMAJ. 2005;172(2):189-94.

30. Tung YC, Chang GM, Chang HY, Yu TH. Relationship between Early Physician Follow-Up and 30-Day Readmission after Acute Myocardial Infarction and Heart Failure. PLoS One. 2017;12(1):e0170061. Published 2017 Jan 27.

31. Siew ED, Peterson JF, Eden SK, et al. Outpatient nephrology referral rates after acute kidney injury. J Am Soc Nephrol. 2012;23(2):305-12. 
32. Kirwan CJ, Blunden MJ, Dobbie H, James A, Nedungadi A, Prowle JR. Critically ill patients requiring acute renal replacement therapy are at an increased risk of long-term renal dysfunction, but rarely receive specialist nephrology follow-up. Nephron. 2015;129(3):164-70.

33. Iorember FM. Malnutrition in Chronic Kidney Disease. Front Pediatr. 2018;6:161. Published 2018 Jun 20.

34. Oluseyi A, Enajite O. Malnutrition in pre-dialysis chronic kidney disease patients in a teaching hospital in Southern Nigeria. Afr Health Sci. 2016;16(1):234-41.

35. Kalender B. Malnutrition in Chronic Kidney Disease and Relationship to Quality of Life. In: Preedy VR, Watson RR, editors. Handbook of Disease Burdens and Quality of Life Measures. New York: Springer; 2010. pp. 3159-70.

36. G SC, L S-M LR-B. What and how much do we eat? 24-hour dietary recall method. Nutr Hosp. 2015 Feb;26(31 Suppl 3):46-8.

37. Hsu CY, Chinchilli VM, Coca S, et al. Post-Acute Kidney Injury Proteinuria and Subsequent Kidney Disease Progression: The Assessment, Serial Evaluation, and Subsequent Sequelae in Acute Kidney Injury (ASSESS-AKI) Study. JAMA Intern Med. 2020;180(3):402-10.

38. Silver S, Adhikari N, Bell C, et al. Nephrologist Follow-Up versus Usual Care after an Acute Kidney Injury Hospitalization (FUSION) [published online ahead of print, 2021 May 21]. Clin J Am SoC Nephrol. 2021;CJN.17331120.

39. Wang AY, Bellomo R, Cass A, et al. Health-related quality of life in survivors of acute kidney injury: The Prolonged Outcomes Study of the Randomized Evaluation of Normal versust Augmented Level Replacement Therapy study outcomes. Nephrology (Carlton). 2015;20(7):492-8.

\section{Figures}




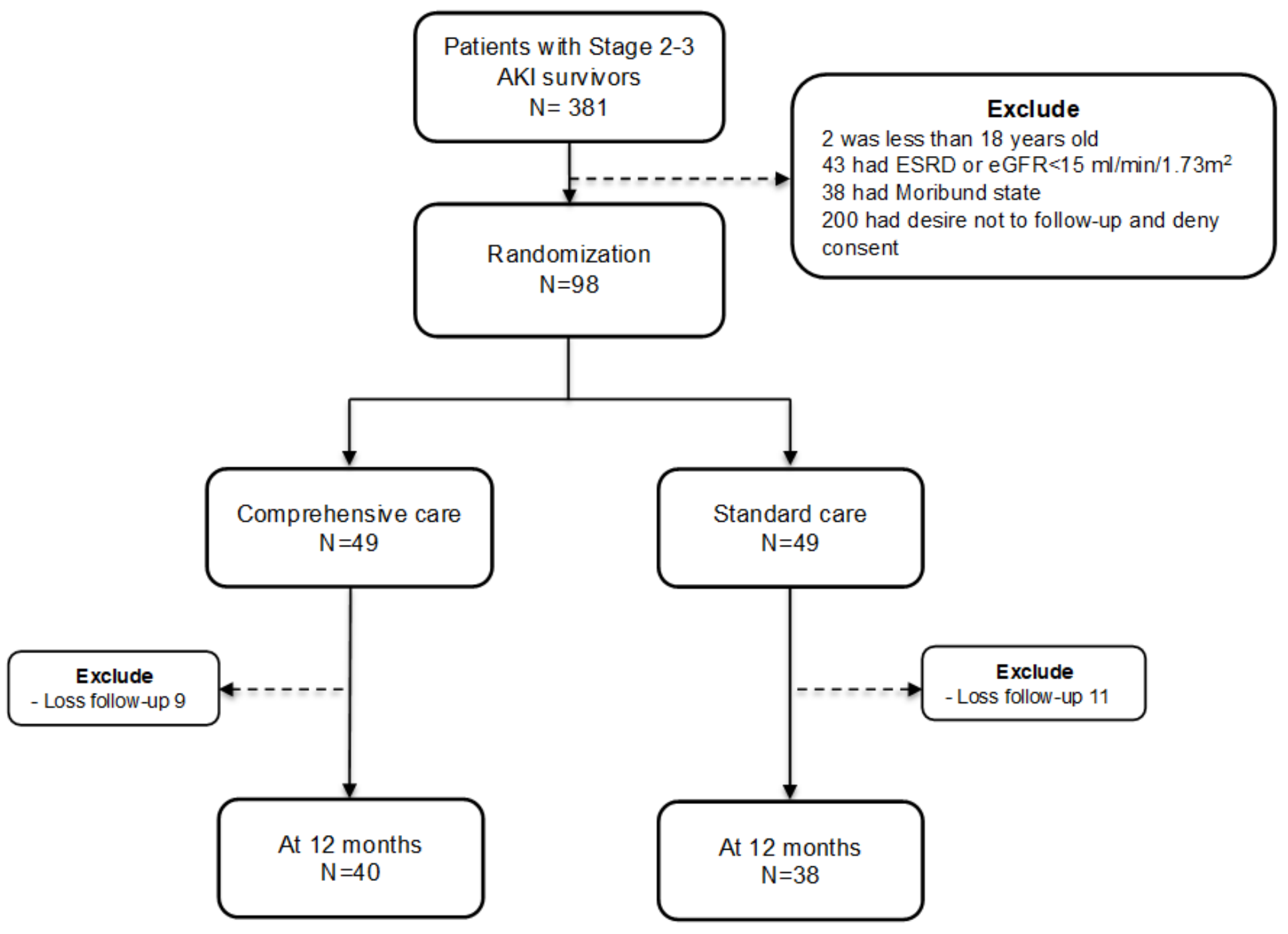

Figure 1

Flow chart of the study Abbreviations: AKI, acute kidney injury; ESRD, end-stage renal disease 


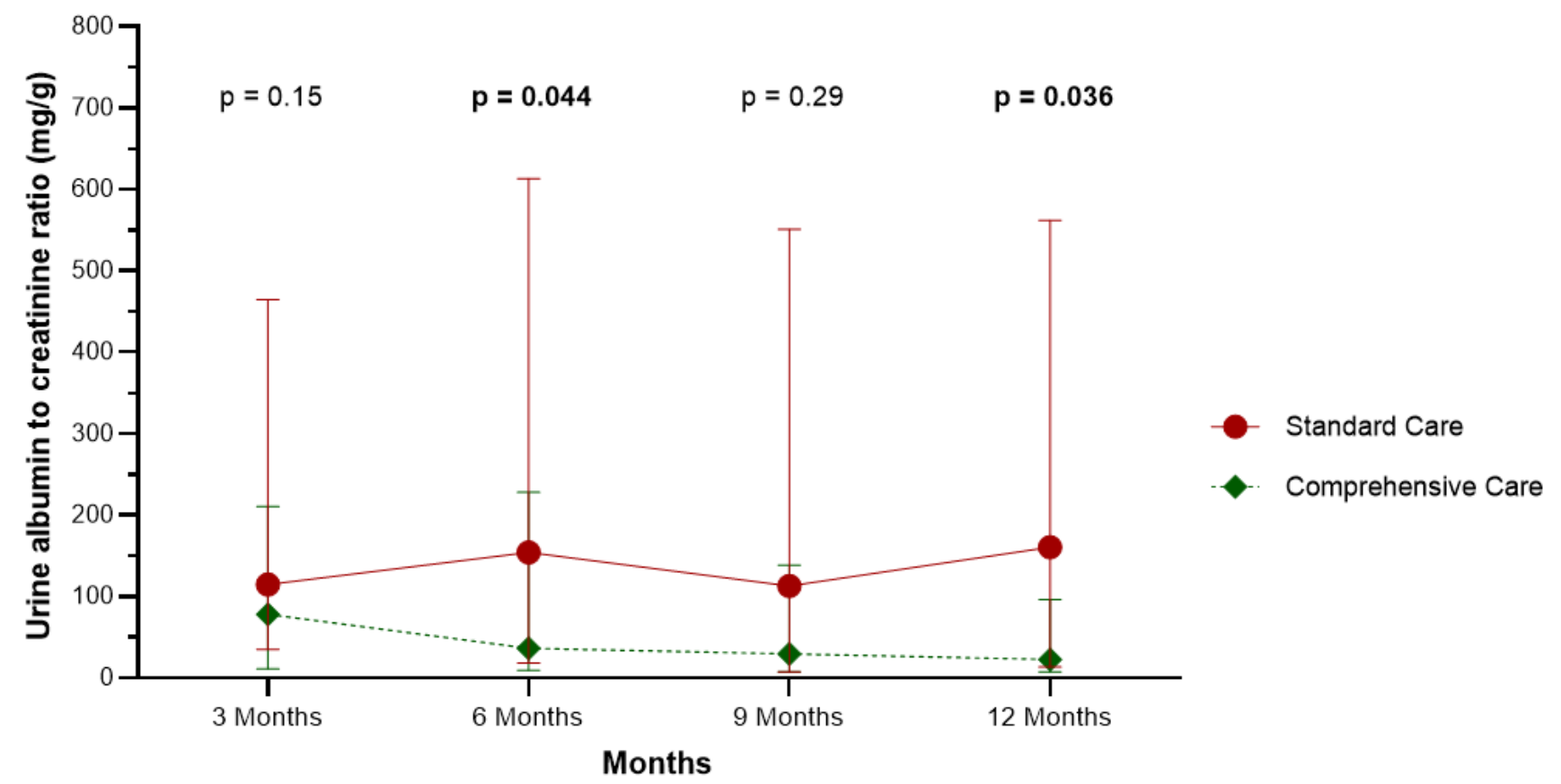

Number of population

$\begin{array}{rllll}\text { Standard care } & 37 & 34 & 30 & 26 \\ \text { Comprehensive care } & 33 & 30 & 27 & 24\end{array}$

Figure 2

Trend in the median UACR Data are shown as the median (IQR) and excluded patients with renal replacement therapy and missing data

\section{Supplementary Files}

This is a list of supplementary files associated with this preprint. Click to download.

- SupplementaryFinalCriticalCare.docx 